\title{
Traumatic Pseudoaneurysm of the Lateral Circumflex Femoral Artery Presenting as Anterior Thigh Compartment Syndrome: A Rare Complication of Intracapsular Hip Fracture Fixation
}

\author{
Avadhoot Kantak
}

\begin{abstract}
Vascular complications after orthopedic surgery are rare. These can have disastrous effects and prompt recognition is of utmost importance. Our case report discusses a rare complication of pseudoaneurysm of the lateral circumflex femoral artery, following fixation of an intracapsular hip fracture. We have reviewed literature and suggested learning points.
\end{abstract}

Keywords: Pseudoaneurysm; Intracapsular hip fracture; Thigh compartment syndrome

\section{Introduction}

Hip fractures are one of the most common fractures that require surgical fixation. The treatment of these fractures is very structured and has clear guidelines issued by organizations like National Institute for Health and Care Excellence (NICE) and Scottish Intercollegiate Guidelines Network (SIGN). Surgery is safe in these fractures and most complications arise as a result of medical morbidities present in this cohort of patients. Vascular complications in these situations are extremely rare [1]. Pseudoaneurysm or false aneurysm results from penetrating vascular injury. It is a re-canalized hematoma which forms between the vessel wall and perivascular soft tissue. This is a rare but serious complication after hip fracture fixation surgery.

\section{Case Report}

A 70-year-old lady presented after an unwitnessed fall at home. She was unable to weight bear and complained of severe left

Manuscript submitted March 28, 2017, accepted April 18, 2017

East Surrey Hospital, 67, Crescent way, Horley, Surrey, UK.

Email: dravadhoot@yahoo.co.uk hip pain. She was brought into the hospital by ambulance. Clinical examination revealed a shortened and externally rotated limb. There was no active or passive movement possible in the left hip. Any attempted movements of the hip were very painful. There was no neurovascular deficit in the lower limb. She did not have any significant medical history and was previously well. She had been independently mobile before the fall. Radiographs confirmed a valgus impacted fracture of the intracapsular neck of femur (Fig. 1). A decision was made to fix the fracture with cannulated cancellous screws. The surgery was carried out within $24 \mathrm{~h}$ of admission. Closed reduction on fixation was achieved with three $6.5 \mathrm{~mm}$ cannulated screws (Fig. 2). The surgery was uneventful and the patient was discharged home on fifth postoperative day.

Three days after discharge, i.e. on the eighth postoperative day, the lady re-presented to accident and emergency with painful swollen thigh. The pain was quite excruciating and stopped the lady from mobilizing. On clinical examination, the thigh was significantly swollen, mid thigh diameter was $15 \mathrm{~cm}$ more than the contra lateral side. Hip rotations were not painful but any attempt to move the knee and straight leg raise were very painful. The thigh was very tight and tender on palpation. The distal pulses were well felt. Blood pressure was 140/90 mm Hg. Neurology of the lower limb was intact. Blood

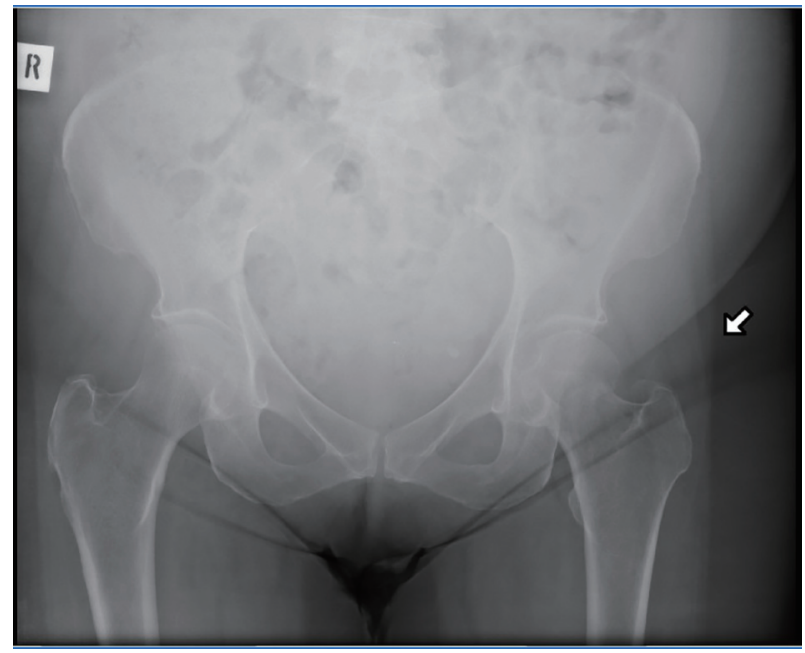

Figure 1. Valgus impacted fracture of femoral neck. 


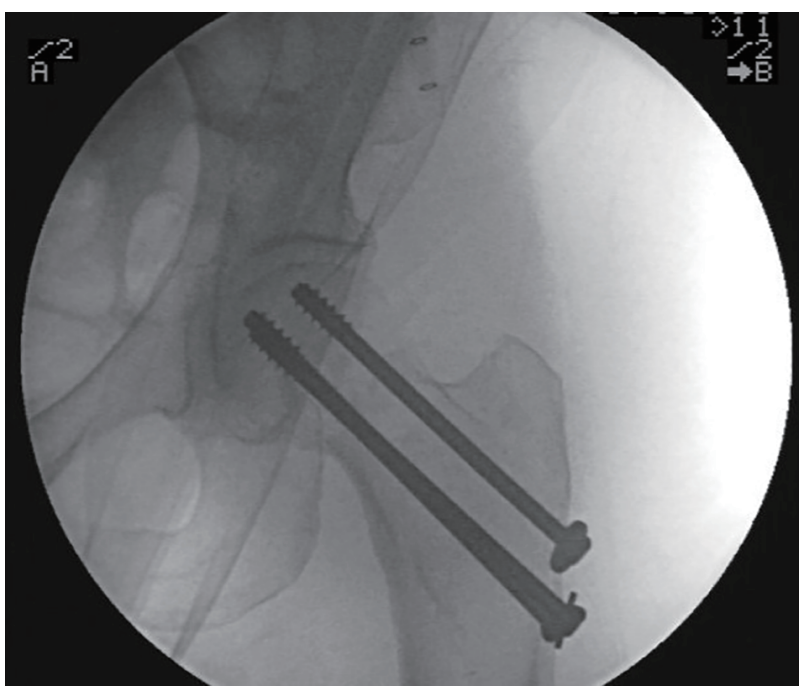

Figure 2. Fixation with cannulated cancellous screws.

tests revealed hemoglobin of $70 \mathrm{~g} / \mathrm{dL}$ (dropped from $98 \mathrm{~g} / \mathrm{dL}$ postoperative), and white cell count was marginally raised at 13. CRP was 60 . Coagulation profile was normal but patient had been on prophylactic anticoagulation as per the guidelines.

Patient was admitted to the ward as a suspected thigh compartment syndrome. Urgent ultrasound (US) scan identified a potential vascular lesion with large anterior thigh compartment hematoma. A CT angiography was performed. This revealed a pseudoaneurysm arising from the lateral circumflex branch of the profunda femoris artery. There was surrounding hematoma causing compartment syndrome (Fig. 3). A plan was made for exploration and compartment release of the anterior thigh compartment. At surgery the anterior thigh compartment was released. Hematoma was evacuated. The pseudoaneurysm

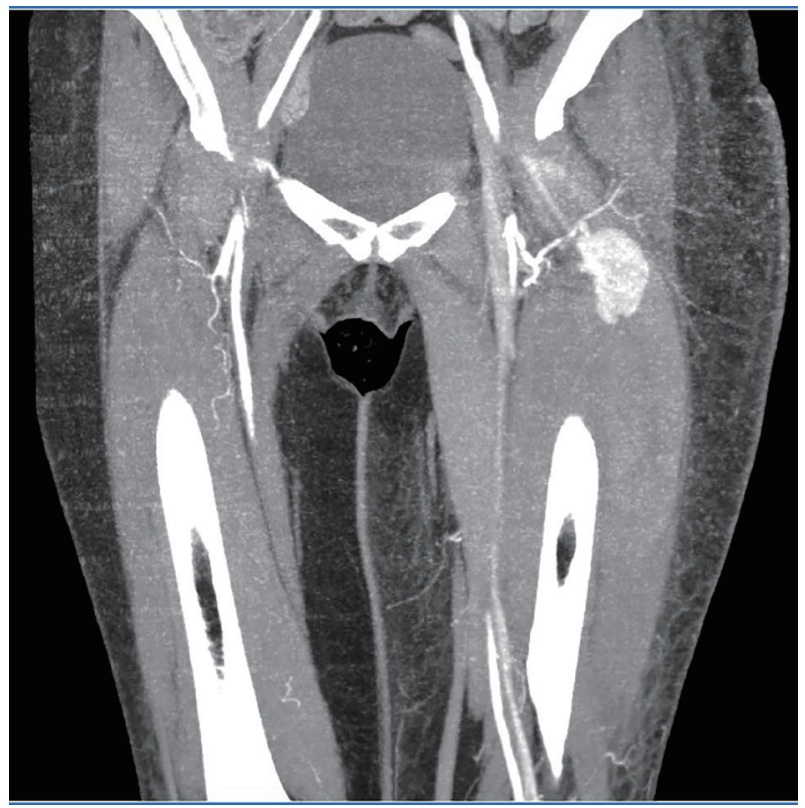

Figure 3. CT angiogram showing the pseudoaneurysm.

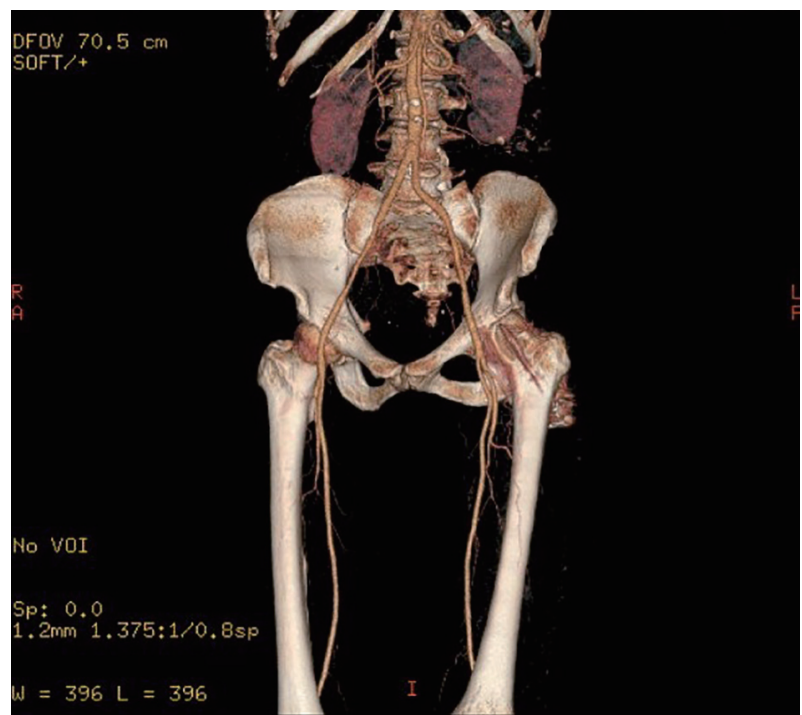

Figure 4. CT angiogram showing complete resolution of the pseudoaneurysm.

was identified; the lateral circumflex femoral artery (LCFA) was isolated and ligated and the pseudoaneurysm was excised. Meticulous hemostasis was achieved. The wound was treated with vacuum assisted closure device for 1 week followed by secondary closure. The patient made a rapid recovery after this procedure. She was discharged home 10 days after the presentation with thigh compartment syndrome. A repeat CT angiogram arranged at 6 weeks after surgery indicated complete resolution of the aneurysm (Fig. 4). The patient has had no concerns regarding her hip and thigh since.

\section{Discussion}

Hip fracture is common, with 65,000 such injuries each year leading to the occupation of over 4,000 inpatient beds at any one time across England, Wales and Northern Ireland [2].

Femoral pseudoaneurysm is an extremely rare complication in orthopedic surgery. According to Karanikas et al [1], it occurred in only three of 1,417 patients undergoing surgery for proximal femoral fractures $(0.21 \%)$. Fixation of these fractures is a procedure requiring moderate operative skills. Most complications arise due to patient factors or technical errors. The first report of pseudoaneurysm was described by Dameron in 1964 [3]. Subsequently there have been quite a few reports of iatrogenic vascular injuries during dynamic hip screw fixation [4-7]. The usual mechanism is over drilling of the far cortices, over penetration of the screws or inadvertent deep placement of retractor clamps. There is also a possibility of arterial puncture due to a displaced bony fragment, either during surgery or at the time of mobilization [5].

\section{Anatomy and probable injury mechanism}

The common femoral artery (CFA) bifurcates into the superfi- 
cial femoral artery (SFA) and profunda femoris artery (PFA). The medial femoral circumflex artery (MFCA) arises from the PFA, winds around the medial aspect of the femur to lie posterior to the femoral neck. The LFCA arises from the lateral side of the PFA; it traverses across iliopsoas, anterior to the femoral neck, running beneath sartorius to lie deep to rectus femoris. It divides into three further branches: the ascending, transverse and descending, one of which was likely to have been injured in our case. The relatively anterior position of the LFCA renders it at-risk from a misplaced wire passed inadvertently along the anterior femoral neck into the soft tissues. The MFCA, primarily, and the ascending branch of LFCA form an extracapsular ring that enters the hip joint capsule, in order to supply the femoral head. These vessels are at the greatest risk of disruption in intracapsular fractures of the neck of femur $[8,9]$.

After a thorough review of literature we found only a few cases of pseudoaneurysm affecting the lateral circumflex femoral artery $[10,11]$. This being complicated by a clinical thigh compartment is even rarer. In our case, the surgery to fix the fracture was a minimally invasive cannulated screw fixation. The risk of vascular injury in such procedures is extremely uncommon. We postulate that the complication in our case was a result of an inadvertent penetration of a guide wire into the soft tissue anterior to the femoral neck, which caused the initial vascular injury to the LCFA. In our opinion, the high risk of injury to LCFA can be attributed to two predisposing factors. Relative immobility of the profunda femoris artery, brought on internal rotation of the leg during reduction manoeuvre, is one and stiffness of the vessel wall due to atherosclerosis is other. We do not think other factors like iatrogenic injury due to retractors, far cortical perforation, displaced fracture spike, etc. had any role in our case, as the procedure was minimally invasive with use of a $5 \mathrm{~cm}$ long incision.

The diagnosis of this complication can be delayed due to difficulty in identifying the symptoms early. The symptoms of thigh swelling, pain, and muscle weakness are quite non-specific. They also coincide with the symptoms of fracture fixation/surgery making it difficult to reach a diagnosis early. The specific symptoms of a bruit over the pseudoaneurysm can be almost impossible to decipher clinically due to the extensive soft tissue cover over the pseudoaneurysm. When there is even a slightest doubt about a vascular injury, we recommend radiological imaging to confirm the diagnosis. A US scan or a Doppler scan is a good initial investigation. It is cheap, easy, portable, non-invasive and reasonably sensitive. US has been reported to have a sensitivity of $94 \%$ and a specificity of $97 \%$ in the detection of postcatheterization pseudoaneurysms [12]. However, the usefulness of US in the evaluation of deep arteries is limited, with low sensitivity. The Doppler has also been found to be less reliable in arterial injuries of the proximal extremities [13]. Moreover, US is operator-dependent, and the evaluation of vessels in trauma patients with fractures or hematomas may be difficult. A CT angiogram remains the gold standard for definitive imaging of arterial trauma [14].

The treatment principles are treatment of hypovolemia and circulatory support, followed by indentifying and controlling the bleeding source and releasing the pressure effects from the hematoma collection. In our case, an open approach was found to be the most suitable because of the acute anterior thigh compartment syndrome. But if the vascular complication has not caused any pressure effects, a non-invasive radio embolization is very successful. Image-guided coil embolization is generally the preferred method of treatment for small to moderate size pseudoaneurysms [15]. However, availability of IR facilities is not definite and in situations where embolization is inaccessible surgical intervention is the preferred treatment. Surgery is indicated for large pseudoaneurysms or those incapable of being embolized. Other modalities of treatment that have been described in the literature include US-guided obliterative compression and direct thrombin injection [16].

In conclusion, our case reports a rare vascular complication which can have quite disastrous outcome if diagnosis is delayed. As surgeons, we should be mindful of non-bony penetration of wires and drills, during any surgical procedure. Any blind soft tissue penetration, howsoever trivial should be avoided. In case of clinical suspicion, a US scan or Doppler scan should be arranged urgently. With regard to early diagnosis, clinical acumen plays a crucial role and a senior review would help to expedite the diagnosis. Every complication teaches us and learning from errors is a medical responsibility. As a response to this complication, we implemented small changes in our practice. We initiated a standard pain score chart for postoperative patients. All patients who were outliers on the pain score were categorically assessed by senior physicians to identify the cause. This helped us in prompt recognition of any complications. All surgeons are encouraged to document any inadvertent soft tissue penetration during surgery. This helps in having a higher index of suspicion whilst managing any complications in these patients.

\section{Conflicts of Interest}

The authors have no conflicts of interest.

\section{Funding}

None.

\section{References}

1. Karanikas I, Lazarides M, Arvanitis D, Papayanopoulos G, Exarchou E, Dayantas J. Iatrogenic arterial trauma associated with hip fracture surgery. Acta Chir Belg. 1993;93(6):284-286.

2. National Hip Fracture Database annual report 2015. Clinical Effectiveness and Evaluation Unit, Royal College of Physicians.

3. Dameron TB Jr. False aneurysm of femoral profundus artery resulting from internal-fixation device (Screw). J Bone Joint Surg Am. 1964;46:577-580.

4. Abraham E, Pankovich AM, Jansey F. False aneurysm of the profunda femoris artery resulting from intertrochanteric fracture. A case report. J Bone Joint Surg Am. 
1975;57(6):871.

5. Fordyce A. False aneurysm of the profunda femoris artery following nail and plate fixation of an intertrochanteric fracture. Report of a case. J Bone Joint Surg Br. 1968;50(1):141-143.

6. Wolfgang GL, Barnes WT, Hendricks GL Jr. False aneurysm of the profunda femoris artery resulting from nail-plate fixation of intertrochanteric fracture. A case report and review of the literature. Clin Orthop Relat Res. 1974(100):143-150.

7. Ebong WW. False aneurysm of the profunda femoris artery following internal fixation of an intertrochanteric femoral fracture. Injury. 1978;9(3):249-251.

8. Gray H. Anatomy of the Human Body. 20th edn. (Online) 2000 www.bartleby.com.

9. Koval KJ. In: Zuckerman JD, editor. Handbook of Fractures. 3rd edn. Philadelphia, PA: LippincottWilliams \& Wilkins; 2006.

10. Kakarala GK, Van Rensburg L, Parker M. Pseudo-aneurysm of the lateral circumflex femoral artery. Eur J Trauma. 2006;32:480-481.

11. Hung CC, Wang CC, Lin LC, Pan RY, Shen HC, Lee CH,
Huang GS. Profunda femoris artery injury following internal fixation with cannulated hip screws for a femoral neck fracture. J Med Sci 2016; 36: 31-34.

12. Morgan R, Belli AM. Current treatment methods for postcatheterization pseudoaneurysms. J Vasc Interv Radiol. 2003;14(6):697-710.

13. Soto JA, Munera F, Morales C, Lopera JE, Holguin D, Guarin O, Castrillon G, et al. Focal arterial injuries of the proximal extremities: helical CT arteriography as the initial method of diagnosis. Radiology. 2001;218(1):188194.

14. Peng PD, Spain DA, Tataria M, Hellinger JC, Rubin GD, Brundage SI. CT angiography effectively evaluates extremity vascular trauma. Am Surg. 2008;74(2):103-107.

15. Singh R, Magu S, Kadian VK, Siwach R, Rohilla RK, Dhir V. Unusual cause of haemorrhage from surgical wound in a child. Strategies Trauma Limb Reconstr. 2009;4(1):45-48.

16. Dillon JP, O'Brien GC, Laing AJ, Adelowokan T, Dolan M. Pseudoaneurysm of the profunda femoris artery following an inter-trochanteric fracture of the femur. Injury Extra. 2004;35(3):30-32. 